\title{
The cholinesterase-like domain of thyroglobulin functions as an intramolecular chaperone
}

\author{
Jaemin Lee,,$^{1,2}$ Bruno Di Jeso, ${ }^{3}$ and Peter Arvan1,2 \\ 1Division of Metabolism, Endocrinology and Diabetes and 2Program of Cellular and Molecular Biology, University of Michigan Medical Center, \\ Ann Arbor, Michigan, USA. 'Laboratorio di Patologia Generale, Dipartimento di Scienze e Tecnologie Biologiche ed Ambientali, \\ Facoltà di Scienze MFN, Università degli Studi di Lecce, Lecce, Italy.
}

\begin{abstract}
Thyroid hormonogenesis requires secretion of thyroglobulin, a protein comprising Cys-rich regions I, II, and III (referred to collectively as region I-II-III) followed by a cholinesterase-like (ChEL) domain. Secretion of mature thyroglobulin requires extensive folding and glycosylation in the ER. Multiple reports have linked mutations in the ChEL domain to congenital hypothyroidism in humans and rodents; these mutations block thyroglobulin from exiting the ER and induce ER stress. We report that, in a cell-based system, mutations in the ChEL domain impaired folding of thyroglobulin region I-II-III. Truncated thyroglobulin devoid of the ChEL domain was incompetent for cellular export; however, a recombinant ChEL protein ("secretory ChEL") was secreted efficiently. Coexpression of secretory ChEL with truncated thyroglobulin increased intracellular folding, promoted oxidative maturation, and facilitated secretion of region I-II-III, indicating that the ChEL domain may function as an intramolecular chaperone. Additionally, we found that the I-II-III peptide was cosecreted and physically associated with secretory ChEL. A functional ChEL domain engineered to be retained intracellularly triggered oxidative maturation of I-II-III but coretained I-II-III, indicating that the ChEL domain may also function as a molecular escort. These insights into the role of the ChEL domain may represent potential therapeutic targets in the treatment of congenital hypothyroidism.
\end{abstract}

\section{Introduction}

Thyroid hormone is essential for development and oxidative metabolism in vertebrates. The synthesis of thyroid hormones involves secretion of thyroglobulin $(\mathrm{Tg})$ to the apical extracellular lumen of follicles that serve as the basic functional unit of the thyroid gland. Once secreted, $\mathrm{Tg}$ is iodinated via the activity of a thyroid-specific peroxidase to form thyroxine (the major thyroid hormone) involving specific iodinated tyrosyl residues within the $\mathrm{Tg}$ polypeptide backbone (1). No other endogenous proteins of the thyroid are capable of serving as precursors for thyroid hormone synthesis, rendering vertebrate organisms completely dependent on structural information encoded in the $\mathrm{Tg}$ molecule - both for directing iodotyrosyl coupling for thyroxine synthesis (2) and for passing ER quality control in the secretory pathway (3). Cumulatively, steps leading to $\mathrm{Tg}$ export from the ER are rate limiting in its overall secretion (4). Peak secretion of Tg, as measured in cell culture, occurs on the order of 3 hours after synthesis (5).

The Tg cDNA initially encodes 3 regions referred to collectively as region I-II-III, that span $80 \%$ of the full-length Tg monomer (2,746 amino acids in mouse $\mathrm{Tg})$, with each region involving multiple Cys-rich repeat domains covalently "stapled" by intradomain disulfide bonds (6); while the final approximately 500 amino acids of Tg are strongly homologous to acetylcholinesterase (7-9). The most important site of thyroxine synthesis involves but a small portion of the molecule at the extreme amino terminus of the $\mathrm{Tg}$ protein $(10,11)$, raising questions about the role of the remaining

Nonstandard abbreviations used: AMS, 4-acetamido-4'-maleimidylstilbene-2,2'disulphonic acid; BFA, brefeldin A; ChEL, cholinesterase-like; Tg, thyroglobulin. Conflict of interest: The authors have declared that no conflict of interest exists. Citation for this article: J. Clin. Invest. 118:2950-2958 (2008). doi:10.1172/JCI35164.
C-terminal 2,250 amino acids (12). Indeed, the overall structure of $\mathrm{Tg}$ remains unknown; nevertheless, it is clear that along its length, $\mathrm{Tg}$ undergoes extensive N-linked glycosylation and monomer folding in the ER (13).

Congenital hypothyroidism with defective $\mathrm{Tg}$ has been reported in species ranging from humans to rats and mice. In all 3 species, the cellular phenotype described includes a dilated ER with induction of ER molecular chaperones (14), activation of ER stress signaling pathways (15), and ER-associated degradation (ERAD) of most of the mutant $\mathrm{Tg}$ gene product (16). Of the cases (and animal models) published to date, the cholinesterase-like (ChEL) domain is a very commonly affected site, involving mutations including the newly described A2215D (17, 18); R2223H (19); G2300D,R2317Q (20); G2355V,G2356R; or the skipping of exon 45 (normally encoding 36 residues of the ChEL domain); as well as Q2638Z mutants (where $Z$ represents stop) (21). In addition are polymorphisms including P2213L and W2482R; and R2511Q that may be associated with an increased incidence of nonmedullary thyroid cancer (22). Congenital hypothyroidism with defective $\mathrm{Tg}$ is caused by the G2300R point mutation localized to the ChEL domain in the $r d w / r d w$ rat $\operatorname{dwarf}(23,24)$, while we identified L2263P as responsible for the hypothyroidism of the $\operatorname{cog} / \operatorname{cog}$ congenital goiter mouse (25). Indeed, a truncated $\mathrm{Tg}$ comprising only region I-II-III and lacking the ChEL domain is blocked within the ER (26). Together, these findings emphasize that in fulllength $\mathrm{Tg}$, a functional ChEL domain is needed to allow $\mathrm{Tg}$ to be efficiently transported to the site of thyroid hormone synthesis, in particular, for exit from the ER.

We show in this study, by contrast, that attaching a signal peptide to the ChEL domain is itself sufficient for rapid and efficient intracellular transport of that protein ("secretory ChEL") to 


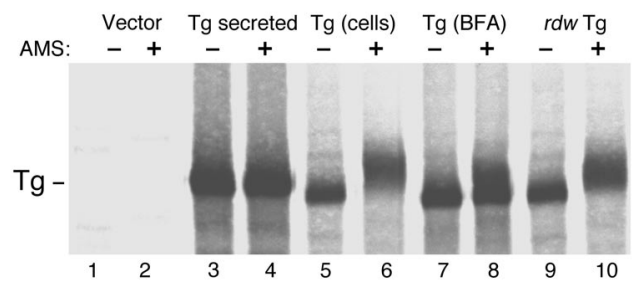

Figure 1

Free Cys thiols in Tg. 293 cells were transiently transfected with an expression vector encoding wild-type $\mathrm{Tg}$, the $r d w \mathrm{Tg}$ mutant (G2300R), or empty vector. At 48 hours after transfection, cells were pulse labeled for 30 minutes with ${ }^{35} \mathrm{~S}$-labeled amino acids and chased for 4 hours in the absence or presence of BFA $(5 \mu \mathrm{g} / \mathrm{ml})$ where indicated. At this time, chase media (lanes labeled "Tg secreted") and cell lysates (all other lanes) were immunoprecipitated with anti-Tg. The immunoprecipitates were denatured in $2 \times$ SDS gel sample buffer lacking reducing agents and mock incubated or incubated with AMS ( $5 \mathrm{mM}, 30^{\circ} \mathrm{C}$ for 1 hour). At the end of the incubation, samples were boiled in the presence of $20 \mathrm{mM}$ DTT and analyzed by $4 \%$ SDS-PAGE and fluorography. A slowed mobility (shift up) of the Tg band after AMS is indicative of free reactive thiols in the Tg molecule that are not apparent in secreted, wild-type $\mathrm{Tg}$.

the extracellular space. Moreover, by physical association with $\mathrm{Tg}$ I-II-III, secretory ChEL rescues in trans maximal protein expression, disulfide maturation, and secretion of Tg I-II-III. Indeed, intentional retention of the functional ChEL domain within the ER, while assisting in Tg I-II-III protein expression and disulfide maturation, prevents its intracellular transport. These data point to both a molecular chaperone and a molecular escort function of the ChEL domain in Tg transport for thyroid hormone synthesis.

\section{Results}

Maturation of recombinant Tg. During folding, Tg must bury 122 free Cys thiols into intrachain disulfide bonds. Reactivity with 4-acetamido-4'-maleimidylstilbene-2,2'-disulphonic acid (AMS) irreversibly alkylates available Cys thiols, increasing by $0.5 \mathrm{kDa}$ the protein

\section{Figure 2}

Arrested disulfide maturation of $\mathrm{Tg}$ bearing a mutation in the ChEL domain. (A) 293 cells were transiently transfected with vector DNA encoding wild-type $\mathrm{Tg}$ (bearing a C-terminal myc-6xHis epitope tag that does not block Tg secretion; ref. 37) or empty vector (control [Con]). Transfected cells were then pulse labeled for 30 minutes with ${ }^{35} \mathrm{~S}$-labeled amino acids and chased at $37^{\circ} \mathrm{C}$ for up to 4 hours in the absence or presence of BFA $(5 \mu \mathrm{g} / \mathrm{ml})$ where indicated. At each chase time, cells were lysed, immunoprecipitated with anti-Tg, and analyzed by nonreducing $4 \%$ SDS-PAGE and fluorography. A region of the gel enriched in disulfide-linked Tg adducts A, B, and C (28) is indicated. Two additional forms of Tg monomer - folding intermediate $D$ and the $\mathrm{E}$ band representing fully oxidized mature $\mathrm{Tg}-$ are shown. (B) Results of an experiment identical to the one represented in $\mathbf{A}$, except that the cells were chased at either $25^{\circ} \mathrm{C}$ (left) or $20^{\circ} \mathrm{C}$ (right). At each time, chase medium was also collected (as indicated), but at these temperatures the media contained no labeled Tg. (C) Results of an experiment identical to the one represented in $\mathbf{A}$, except that cells were transfected with plasmid DNA encoding cog (L2263P) or $r d w$ (G2300R) mutations within the $\mathrm{Tg}$ ChEL domain, and the cell lysis buffer included $20 \mathrm{mM} \mathrm{N}$-ethylmaleimide. No detectable $\mathrm{Tg}$ was secreted, so analysis of the supernatant is not shown. molecular mass per alkylated Cys (27), which can ultimately be detected as a molecular mass shift by SDS-PAGE under reducing conditions. Upon denaturation, Tg secreted from cells (which had already passed ER quality control and received Golgi carbohydrate modifications) showed no detectable reactivity with $5 \mathrm{mM}$ AMS (Figure 1, compare lanes 3 and 4). By contrast, newly synthesized intracellular Tg residing primarily in the ER (5) showed substantial AMS reactivity (compare lanes 5 and 6). Cells treated with brefeldin A (BFA; $5 \mu \mathrm{g} / \mathrm{ml}$ ) accumulated in the ER newly synthesized $\mathrm{Tg}$ that, in terms of AMS reactivity, seemed equivalent to the sum of secreted and intracellular Tg obtained from untreated cells, revealing the ability to discriminate between distinct folded states. In the case of the $r d w$ mutant bearing the G2300R mutation within the ChEL domain of $\mathrm{Tg}$, none of the Tg molecules in the population appeared able to properly mature, as judged by fact that they buried their Cys thiols (Figure 1). By 4\% SDS-PAGE, a mobility shift of this magnitude (above that of the $330-\mathrm{kDa} \mathrm{Tg}$ band) suggests a molecular mass addition of 10-30 kDa, which might potentially represent modification of as many as 20-60 of the first 116 Cys residues of Tg. By contrast, the ChEL domain bearing the mutation has only 6 Cys residues - even modification of all of these residues would amount to a net molecular mass addition of only $3 \mathrm{kDa}$, which would not be expected to be seen as a significant mobility shift of the Tg band. Thus, these data imply that a mutation within the ChEL domain may impact on the ability to efficiently form disulfide bonds within upstream Tg region I-II-III, which contain the majority of Tg Cys residues.

Recent studies have shown that for a brief period after synthesis, Tg newly synthesized by thyrocytes can be detected in mixed disulfides with a number of ER oxidoreductases: adducts known as forms A, B, and C that are detected by nonreducing SDS-PAGE (28). Herein, we used nonreducing SDS-PAGE to examine disulfide maturation of recombinant mouse Tg upon transient expression in 293 cells. For wild-type Tg, adducts in the A, B, and C

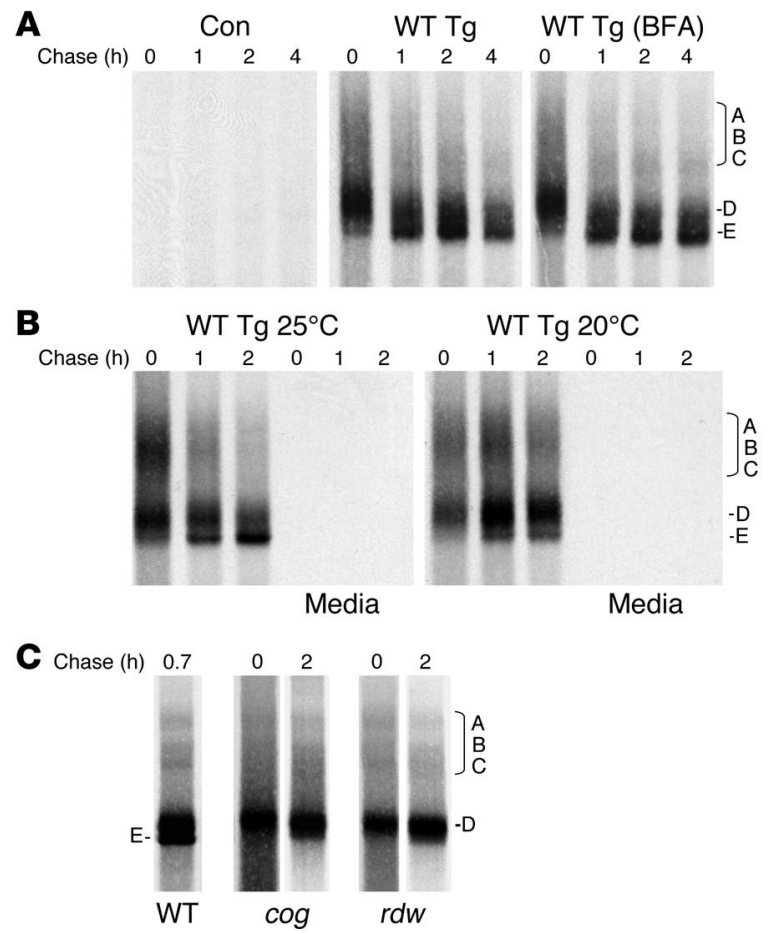




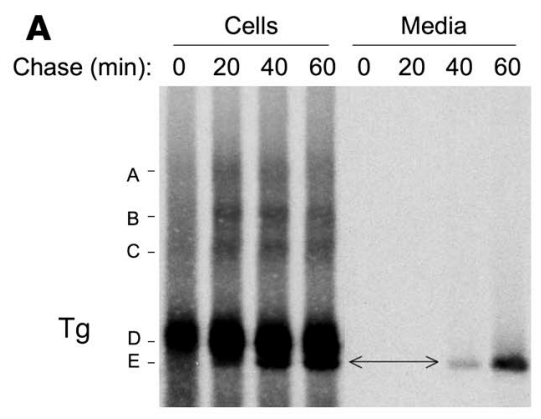

Cell Medium

60

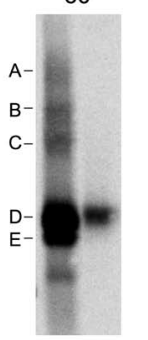

No PNGase F

B
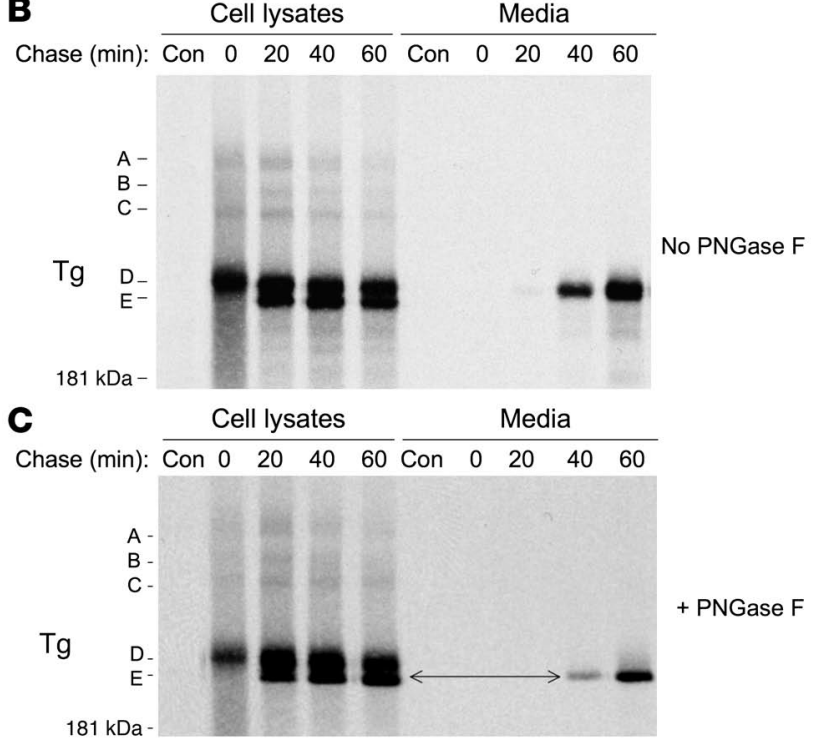

region of the gel (like those reported for endogenous Tg; ref. 28) could be seen, while an incompletely oxidized $\mathrm{Tg}$ monomer called band $\mathrm{D}$ was already the major species at the 0 chase time (Figure $2 \mathrm{~A}$, middle panel). By 1 hour of chase, the predominant $\mathrm{Tg}$ form was already a fully oxidized monomer called band $\mathrm{E}$ (Figure 2A). Because secretion was ongoing, mature $\mathrm{Tg}$ was lost from these cells (data not shown). Under conditions where all forms of $\mathrm{Tg}$ were retained in the ER by treatment of cells with BFA, a quantitative profile of oxidative $\mathrm{Tg}$ folding intermediates could be observed (BFA; Figure 2A, right).

Using PC $\mathrm{Cl} 3$ thyrocytes expressing endogenous $\mathrm{Tg}$, we found that the newly identified $\mathrm{E}$ isoform is essentially identical to that secreted to the medium. What confounds the nonreducing SDS-PAGE analysis of Tg band mobility is the presence of Golgimodified $\mathrm{N}$-linked glycans on $\mathrm{Tg}$ (Figure 3A, right panel). Thus, a comparison of band mobility upon nonreducing SDS-PAGE can be made only after treatment with PNGase F to remove all $\mathrm{N}$-linked oligosaccharides (Figure 3A, left panel; a double-headed arrow establishes the identity of the $\mathrm{E}$ isoform in cells and media). Similar results were obtained for recombinant $\mathrm{Tg}$ expressed in 293 cells when examined without (Figure 3B) or with (Figure 3C) prior PNGase F digestion.

$\mathrm{Tg}$ monomer folding is temperature dependent (29), and $\mathrm{Tg}$ secretion is profoundly inhibited at both $20^{\circ} \mathrm{C}$ and $25^{\circ} \mathrm{C}$. At $25^{\circ} \mathrm{C}$, there was a slight increase in $\mathrm{Tg}$ adducts $\mathrm{A}, \mathrm{B}$, and $\mathrm{C}$ at the 0 chase time (Figure 2B, left). At $20^{\circ} \mathrm{C}$ there was additional prolongation of $\mathrm{Tg}$ adducts and further inhibition of $\mathrm{Tg}$ oxidative maturation from

\section{Figure 3}

Oxidation state of secreted Tg. At each chase time, cells were lysed in buffer including $20 \mathrm{mM} \mathrm{N}$-ethylmaleimide, and the lysates and chase media immunoprecipitated with anti-Tg and analyzed by nonreducing 4\% SDS-PAGE and fluorography. (A) PC Cl3 cells were pulse labeled for 10 minutes with ${ }^{35}$ S-labeled amino acids and then chased for the times indicated. Immunoprecipitates were either undigested or digested with PNGase F as indicated. Folding intermediates A, B, and $C$, which have been characterized in previous studies (28), are shown. Also identifed are 2 closely spaced Tg disulfide isomer bands labeled $\mathrm{D}$ and $\mathrm{E}$, respectively. Because of glycosylation differences, the mature $\mathrm{E}$ isoform does not comigrate with $\mathrm{Tg}$ secreted to the medium at 1 hour of chasing. After PNGase $\mathrm{F}$ digestion to remove $\mathrm{N}$-glycans, all Tg forms exhibit a faster (shifted-down) mobility. Under these conditions, it is now apparent that secreted Tg comigrates with the intracellular $E$ isomer, identifying the most oxidized band as the most mature folded form of Tg. (B) Results of an experiment identical to the one represented in $\mathbf{A}$, except using recombinant $\mathrm{Tg}$ expressed in 293 cells, without PNGase F digestion. (C) Results of a repeat experiment of that shown in panel $\mathbf{B}$, but including PNGase F digestion. The position of a 181-kDa prestained molecular weight standard is shown at left.

folding intermediate $\mathrm{D}$ to the mature $\mathrm{E}$ form (Figure $2 \mathrm{~B}$, right). With these results serving as positive controls for $\mathrm{Tg}$ folding, we proceeded to examine the oxidative maturation of both $\operatorname{cog} \mathrm{Tg}$ and $r d w$ $\mathrm{Tg}$ (which bear mutations in the ChEL domain). As shown in Figure 2C, these proteins, even at 2 hours after synthesis, appeared arrested as early folding intermediates, including both Tg adducts and the incompletely oxidized $\mathrm{D}$ isoform. While we do not yet know the specific $\mathrm{Tg}$ Cys residues involved in either adduct formation or maturation from $\mathrm{D}$ to $\mathrm{E}$ forms, these data further imply that function of the ChEL domain is crucial to global folding of the Tg molecule.

The isolated ChEL domain folds autonomously in the secretory pathway. The Tg ChEL domain exhibits substantial primary sequence simi-

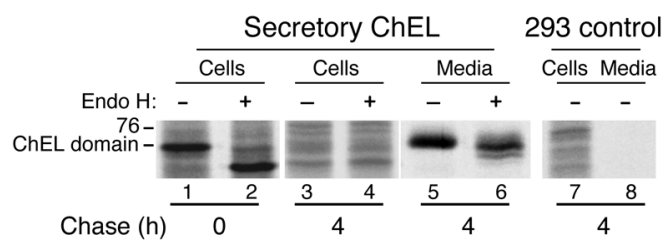

\section{Figure 4}

Efficient exit of the isolated Tg ChEL domain from the ER. 293 cells were transiently transfected with a plasmid encoding the wild-type mouse $\mathrm{Tg}$ ChEL domain preceded by the prolactin signal peptide (Secretory ChEL) or were untransfected (293 control). Cells were pulse labeled for 30 minutes with ${ }^{35}$ S-labeled amino acids and chased for 0 or 4 hours as indicated, at which time the cells were lysed and both lysates and media immunoprecipitated with a rabbit polyclonal anti-Tg. Immunoprecipitates from transfected cells were divided in 2 equal portions and either mock digested or digested with endoglycosidase $\mathrm{H}$ (Endo $\mathrm{H}$ ). Finally, all samples were analyzed by reducing $5.5 \%$ SDS-PAGE and fluorography. The band shift observed after digestion of secretory ChEL from the 0 chase time (shift down, lane 2) is indicative of endoglycosidase $\mathrm{H}$ sensitivity and defines $\mathrm{ChEL}$ that has not yet reached the Golgi complex; in contrast, none of the secreted ChEL shows the same endoglycosidase $\mathrm{H}$ sensitivity, indicating intracellular transport via the Golgi complex. The lanes shown were all run on the same gel, although they are presented noncontiguously. The position of the 76-kDa molecular mass standard is shown at left. 
$\mathbf{A}$
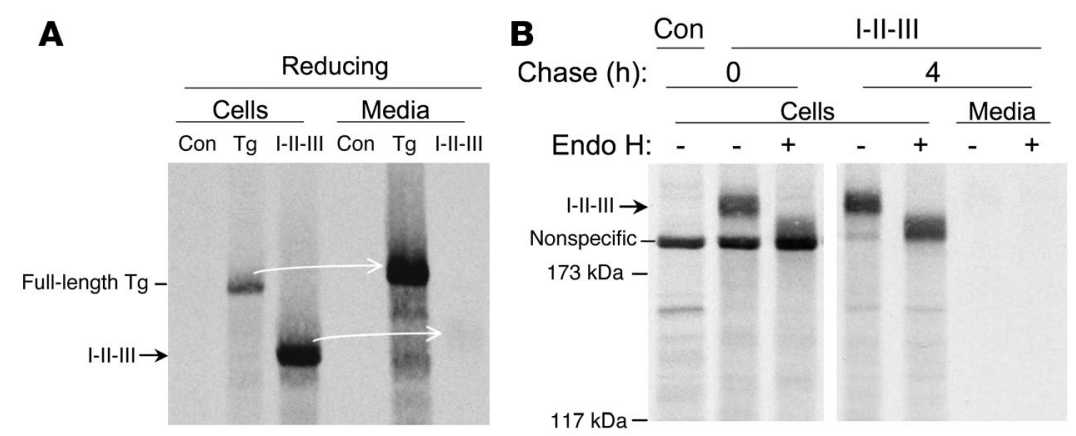

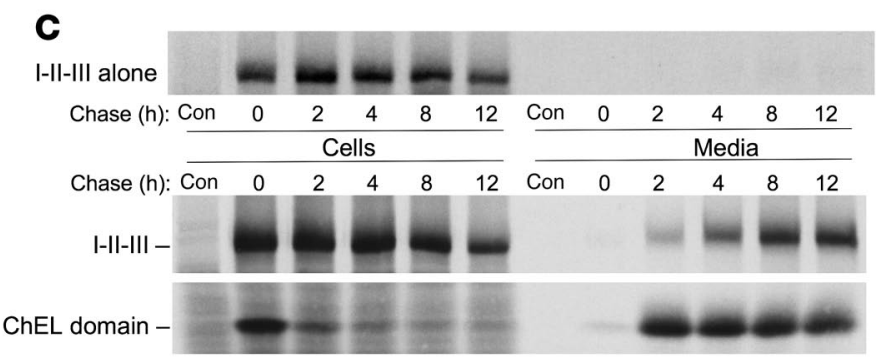

\section{Figure 5}

ER exit of Tg region I-II-III in the absence and presence of ChEL domain. (A) 293 cells were either untransfected (control) or transiently transfected with $2 \mu \mathrm{g}$ plasmid DNA encoding either full-length wild-type mouse Tg or Tg region I-II-III. Cells were pulse labeled and chased and samples prepared and immunoprecipitated with anti-Tg as in Figure 4, with analysis by reducing 4\% SDS-PAGE and fluorography. Arrows highlight secretion (or lack thereof) from cells to media. (B) The first lane represents untransfected control cells. The remaining cells transfected to express Tg region I-II-III were pulse labeled as in $\mathbf{A}$ and then lysed without or with 4 hours of chasing. Tg immunoprecipitates from cell lysates and media were either mock digested or digested with endoglycosidase $\mathrm{H}$ for 1 hour at $37^{\circ} \mathrm{C}$, before reducing $4 \%$ SDS-PAGE and fluorography. Lanes were run contiguously but have been separated for clarity. The positions of molecular mass standards are shown at left. (C) Lower panels: 293 cells were transiently transfected with $0.5 \mu \mathrm{g}$ plasmid DNA encoding I-II-III cotransfected with $2.5 \mu \mathrm{g}$ of plasmid DNA encoding the secretory ChEL domain or untransfected controls. Cells were pulse labeled for 30 minutes with ${ }^{35} \mathrm{~S}$-labeled amino acids and chased for the times indicated. At each chase time, the cells were lysed and both lysates and media immunoprecipitated with polyclonal anti-Tg to recover both I-II-III and secretory ChEL proteins, as revealed by reducing 5.5\% SDS-PAGE and fluorography. The upper panel represents a negative control with identical transfection of I-II-III but without cotransfection of the secretory ChEL plasmid.

larity with authentic acetylcholinesterase, a well-studied protein (30) that is fully competent for intracellular transport to the cell surface. We therefore attempted to examine secretory behavior of the isolated ChEL domain driven into the ER via an artificial (prolactin) signal peptide. Indeed, within 4 hours after synthesis, the recombinant secretory ChEL protein was nearly completely released from cells as an endoglycosidase $\mathrm{H}$-resistant species (Figure 4, lane 6), indicating that it traversed the intracellular transport pathway as a normal secretory protein.

The ChEL domain is an intramolecular chaperone for Tg region I-II-III. We previously reported that Tg region I-II-III is secretion incompetent (26). To confirm this point, we followed the secretion and sensitivity to endoglycosidase $\mathrm{H}$ digestion of newly synthesized I-II-III 4 hours after synthesis. Indeed, while full-length wild-type Tg was efficiently secreted, I-II-III remained intracellular (Figure $5 \mathrm{~A})$. Persistence of sensitivity to endoglycosidase $\mathrm{H}$ digestion (Figure $5 \mathrm{~B}$ ) indicated that I-II-III does not have sufficient information for efficient intracellular transport. This was also true even at extended chase times (Figure 5C, upper panel). These data support our previous hypothesis that the ChEL domain may be required for normal conformational maturation and intracellular transport of $\operatorname{Tg}(26)$.

To better understand the relationship of the I-II-III domains to the ChEL domain, we coexpressed these as separate proteins within the ER. Remarkably, in trans, presence of the secretory ChEL domain in the ER rescued the export of I-II-III. This secretion was progressive over time (Figure 5C, lower panels).

A likely implication of these findings is that I-II-III directly interacts with the ChEL domain. To examine this, we constructed a secretory ChEL with a carboxyterminal myc-epitope tag. Then either secretory ChEL or secretory ChEL-myc was coexpressed with I-II-III in 293 cells. After metabolic labeling, the chase medium bathing these cells was collected and evenly divided for immunoprecipitation with either anti-Tg or anti-myc (Figure 6A). Anti-Tg (lanes 1-3) directly immunoprecipitated both secretory ChEL (or ChEL-myc) as well as the rescued cosecreted I-II-III. Anti-myc (lanes 4-6) could not immunoprecipitate untagged ChEL and also failed to recover any I-II-III. However, anti-myc immunoprecipitated secretory ChEL-myc and quantitatively coprecipitated all of the I-II-III (Figure 6A, lane 6) that could be directly immunoprecipitated with polyclonal anti-Tg (lane 3).

These data strongly suggest that nearly $100 \%$ of secreted I-II-III associates with secretory ChEL within the ER and remains associated throughout the secretory pathway. Yet newly synthesized I-II-III is secreted with much slower kinetics than the cohort of labeled ChEL molecules synthesized at the same time (Figure 5C). I-II-III secretion must be dependent upon subsequently synthesized ChEL molecules, as post-pulse inhibition of further protein synthesis with cycloheximide (Figure 6B, lanes 3 and 4) blocked secretion of newly synthesized I-II-III even as it had no effect on the 


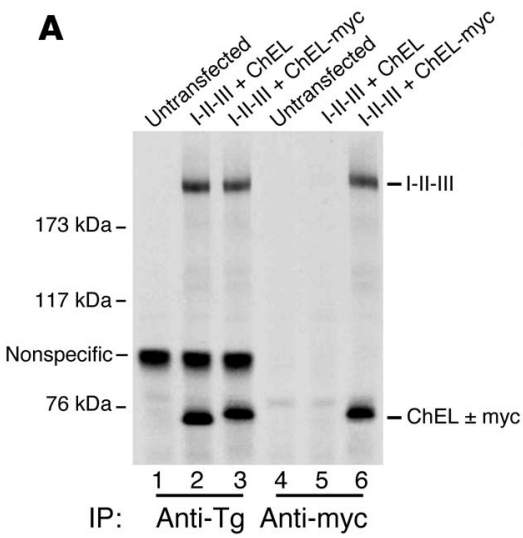

\section{B}

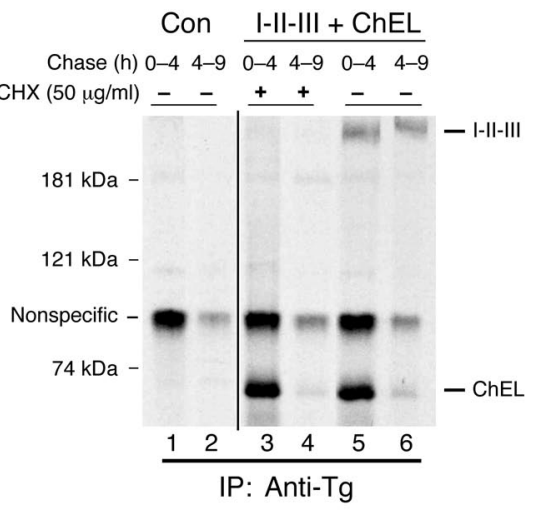

\section{Figure 6}

Secreted I-II-III protein is physically associated with secretory ChEL protein. (A) 293 cells were transiently transfected with $0.5 \mu \mathrm{g}$ plasmid DNA encoding I-II-III and cotransfected with $2.5 \mu \mathrm{g}$ of plasmid DNA encoding the secretory ChEL domain either lacking or containing a myc epitope tag, as indicated. The cotransfected cells or untransfected controls were pulse labeled for 30 minutes and chased in complete media, and the secretion after 6 hours was analyzed by immunoprecipitation with anti-Tg or anti-myc. Immunoprecipitates and coprecipitates were analyzed by reducing 5.5\% SDS-PAGE and fluorography. Addition of the myc tag slightly retards the SDS-PAGE mobility of the ChEL domain. Note that anti-myc precipitation of ChEL-myc coprecipitates I-II-III. (B) Cells untransfected (control) or cotransfected and pulse labeled as in A (I-II-III $+\mathrm{ChEL}$ ) were chased in complete media for the time intervals shown, in the presence or absence of cycloheximide (CHX). The media were immunoprecipitated with anti-Tg and analyzed by reducing SDS-PAGE and fluorography. These lanes were run contiguously; a black line has been added for clarity to separate the samples. Note that prelabeled ChEL secretion proceeded rapidly in the presence of $\mathrm{CHX}$, but prelabeled I-II-III secretion was blocked. The positions of molecular mass markers are shown at left.

secretion of labeled ChEL. Thus, I-II-III is not ready to interact with ChEL when I-II-III is first made but evidently needs preliminary folding to acquire competence for ChEL interaction. By contrast, ChEL export can proceed independently of I-II-III (Figure 4), decreasing its availability in the ER, which may limit efficiency of I-II-III transport (Figure 5C). To test this, we performed cotransfections of cDNAs encoding I-II-III and secretory ChEL at different plasmid ratios (with I-II-III cDNA and total DNA per transfection held constant). As shown in Figure 7A, increasing secretory ChEL cDNA increased the expression and secretion of the ChEL protein (bottom panel). Simultaneously, this also increased the quantity of I-II-III that was recovered intracellularly after a 6-hour chase, despite the fact that the transfection quantity of I-II-III cDNA was held constant (upper panel). As the initially synthesized I-II-III (0 chase time) was only marginally affected by coexpression of secretory ChEL (Figure 7B), it appears that most of the intracellular increase in I-II-III protein reflects stabilization after synthesis. Indeed, we determined that total I-II-III recovery was increased $100 \%$ (i.e., doubled) at 16 hours of chase when secretory ChEL was expressed compared with vector DNA alone (Figure $7 \mathrm{~B}$ ).
A

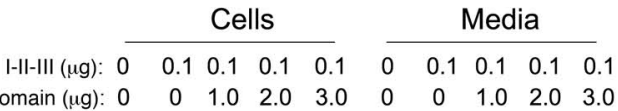

ChEL domain $(\mu \mathrm{g}): 0 \quad 0 \quad \begin{array}{lllllllll}0 & 1.0 & 2.0 & 3.0 & 0 & 0 & 1.0 & 2.0 & 3.0\end{array}$

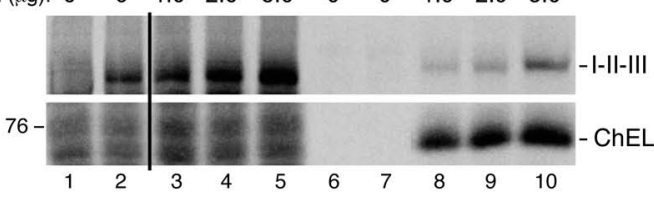

B

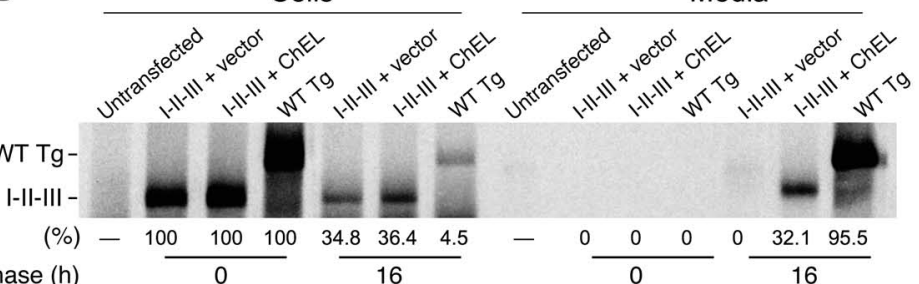

Figure 7

ChEL interaction improves recovery as well as secretion of Tg I-II-III. (A) 293 cells were triply transfected with empty vector plus a plasmid encoding Tg region I-II-III (always $0.1 \mu \mathrm{g}$ DNA per well) plus a plasmid encoding the secretory ChEL domain (at different DNA levels as shown). DNA in each transfection totaled $3.1 \mu \mathrm{g}$ per well. Transfected cells were pulse labeled for 30 minutes with ${ }^{35} \mathrm{~S}$-labeled amino acids and chased for 6 hours, at which time the cells were lysed, and both lysates and media were immunoprecipitated with anti-Tg and analyzed by reducing $5.5 \%$ SDS-PAGE and fluorography, as shown. The position of a $76-\mathrm{kDa}$ molecular mass marker is shown at left. The figure has been spliced at the position indicated by a black line (between lanes 2 and 3), but all data were derived from a single exposure of the same gel. (B) Cells were either untransfected or transfected with $0.5 \mu \mathrm{g}$ plasmid DNA encoding I-II-III plus $2.5 \mu \mathrm{g}$ of vector DNA or that encoding secretory ChEL. As a positive control, $2 \mu \mathrm{g}$ of plasmid DNA encoding wild-type Tg was transfected in parallel. Cells were pulse labeled for 30 minutes with ${ }^{35} \mathrm{~S}-\mathrm{labeled}$ amino acids and chased for either 0 or 16 hours, at which time the cells were lysed and both lysates and media immunoprecipitated with anti-Tg and analyzed by reducing 5.5\% SDS-PAGE and phosphorimaging, as shown. The intracellular band density at the 0 chase time was defined as $100 \%$; based on this, the recovery of each band at 16 hours is shown. Total recovery of labeled I-II-III alone at 16 hours was approximately $35 \%$, while total recovery of labeled I-II-III (cells plus media) in the presence of secretory ChEL was approximately $68 \%$. 

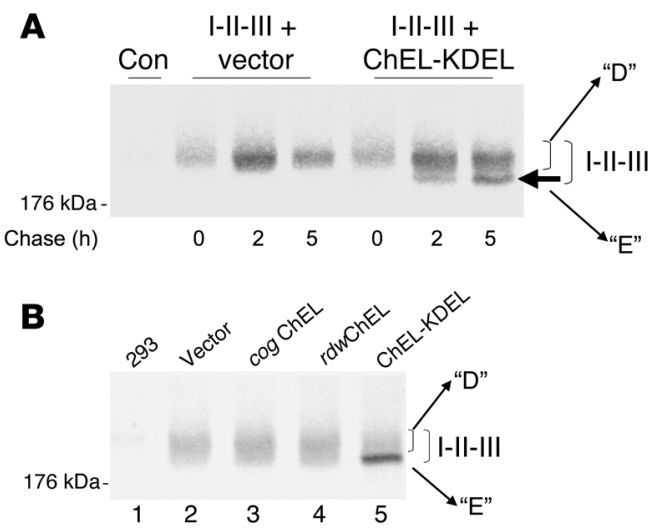

Figure 8

ChEL functions as a molecular chaperone for I-II-III. (A) Cells were either untransfected (control) or transfected with a plasmid encoding I-II-III plus either empty vector or secretory ChEL-KDEL, as indicated. The transfected cells were pulse labeled for 30 minutes with ${ }^{35} \mathrm{~S}$-labeled amino acids and chased for the times indicated. At each chase time, cells were immunoprecipitated with anti-Tg and newly synthesized I-II-III analyzed by nonreducing 4\% SDS-PAGE and fluorography. Absence of recovery of I-II-III from untransfected control cells is shown at left. The band seen at time 0 and after chasing for 2 hours appears equivalent to that of the $\mathrm{D}$ isoform of full-length $\mathrm{Tg}$. In the presence of the ChEL domain in the ER, a faster-migrating band equivalent to that of the mature $E$ isoform of full-length $\mathrm{Tg}$ is detected (filled arrow). (B) Results of an experiment using the same cotransfection protocol and analysis as in $\mathbf{A}$, except the second plasmid is either vector alone or secretory ChEL domain containing the cog mutation, the $r d w$ mutation, or the KDEL appendage. Absence of recovery of I-II-III from untransfected 293 cells is shown in lane 1. The position of a $176-k D a$ molecular mass marker is shown at left.

Accompanying the increased recovery in I-II-III was an increase in the amount of I-II-III secretion (Figure 7A).

If the ChEL domain were acting as a molecular chaperone for I-II-III, we reasoned that secretory ChEL might be able to enhance oxidative folding of I-II-III. To test this, we designed a secretory ChEL-KDEL construct, in which the KDEL sequence retains the ChEL domain in the early secretory pathway (discussed below). In a pulse-chase format, presence of the ChEL-KDEL protein promoted disulfide maturation of I-II-III from an incompletely oxidized intermediate (reminiscent of intermediate D of full-length Tg; see Figure 2) to a more mature (E-like) form (Figure 8A, arrows). To determine whether ChEL domain tertiary structure is required for oxidative rescue of I-II-III, we compared the ChEL-KDEL construct with other secretory ChEL constructs bearing $\operatorname{cog}$ or $r d w$ mutations, which are also retained within the early secretory pathway. As shown in Figure 8B, unlike ChEL-KDEL, these mutations within the ChEL domain had little or no beneficial effect on oxidative maturation of I-II-III. By contrast, authentic secretory ChEL (without KDEL) promoted secretion of I-II-III protein that, after deglycosylation with PNGase F, exhibited oxidative maturation comparable to that of the mature $\mathrm{E}$ form seen intracellularly (Figure 9). Together, the data in Figures 8 and 9 strongly suggest that a native ChEL domain acts like an intramolecular chaperone, facilitating oxidative maturation of upstream $\mathrm{Tg}$ domains.

The ChEL domain is a molecular escort for Tg region I-II-III. If the ChEL domain serves only as a true molecular chaperone within $\mathrm{Tg}$, then we would expect that when expressed in trans, secretory ChEL (like other molecular chaperones) would show a binding preference for early I-II-III folding intermediates over fully mature molecules. To examine this, we coimmunoprecipitated intracellular I-II-III with secretory ChEL-myc in cells treated with BFA to block intracellular transport in the secretory pathway. In the absence of ChEL, I-II-III exhibited defective oxidative maturation (Figure 10, lanes 2-4), while in the presence of secretory ChEL-myc, anti-Tg directly and efficiently immunoprecipitated both immature D and mature E forms of I-II-III (lanes 5-7). The presence of ChEL appeared to increase recovery of I-II-III, consistent with results shown in Figure 7. By contrast, the immunoprecipitation of ChEL with anti-myc seemed inefficient at coprecipitation of the unfolded I-II-III isomer, as compared with the efficient recovery of the faster-migrating, mature I-II-III (Figure 10, lanes 9 and 10). These data along with the persistent ChEL association with I-II-III even after secretion (Figure 6A) indicate that ChEL serves as more than an intramolecular chaperone for unfolded $\mathrm{Tg}$ molecules. If indeed the ChEL domain helps to physically convey I-II-III export from the ER, then if ChEL transport were to be disrupted, I-II-III transport would be disrupted in parallel. This proved to be the case, as unlike secretory ChEL, secretory ChEL-KDEL permitted secretion of I-II-III only to the extent that the ChEL-KDEL itself escaped from the cells (Figure 11, A and B), despite the fact that ChEL-KDEL facilitated the oxidative maturation of I-II-III (Figure 8). As predicted by results in Figure 8B, secretory ChEL bearing $\operatorname{cog}$ or $r d w$ mutations also did not support I-II-III secretion (Figure 11B), just as these mutations do not permit secretion of intact $\mathrm{Tg}$. Together, the data indicate that the ChEL domain functions as both an intramolecular chaperone and molecular escort for Tg maturation and transport in the secretory pathway.

\section{Discussion}

$\mathrm{Tg}$ has evolved as the unique protein precursor for thyroid hormone synthesis. Specially encoded structural features for thyroid hormone formation and iodide storage (2) are known to come into play once the $\mathrm{Tg}$ molecule has been delivered extracellularly, where it comes in contact with the thyroidal iodination machinery (31). However, structural features encoding $\mathrm{Tg}$ transport in the secretory pathway - required for $\mathrm{Tg}$ to serve as a prohormone - have

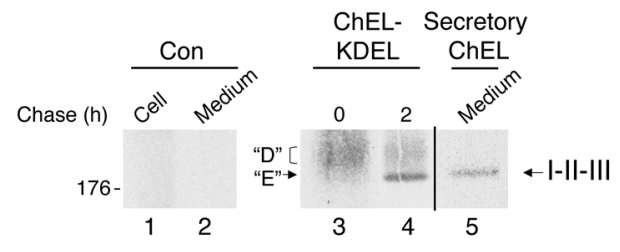

\section{Figure 9}

Oxidation state of secreted I-II-III. 293 cells were transfected either with empty vector (control) or with secretory ChEL bearing or lacking the KDEL appendage. The cells were pulse labeled for $30 \mathrm{~min}$ with ${ }^{35} \mathrm{~S}$ labeled amino acids and then chased for the times indicated (control cells were chased for 2 hours). The cells were lysed, and both lysates and chase medium immunoprecipitated with anti-Tg and subjected to PNGase F digestion to remove N-glycans. The samples were then analyzed by nonreducing 5.5\% SDS-PAGE. Under these conditions, it is apparent that secreted I-II-III closely migrates with the intracellular E isomer of I-II-III. A black line has been added to distinguish the medium from the cell lysate. The position of a 176-kDa molecular mass marker is shown at left. 


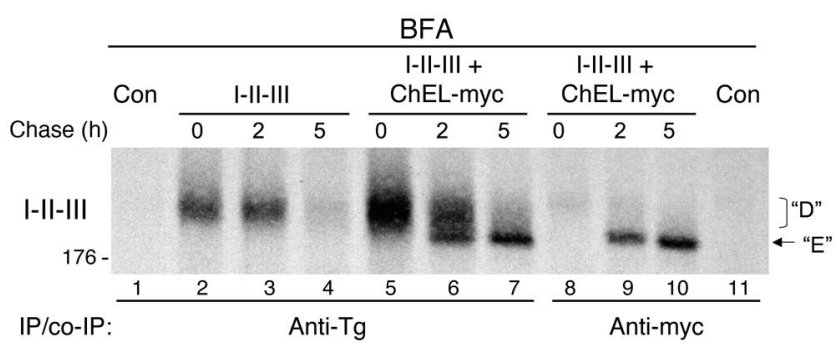

\section{Figure 10}

Intracellular association of secretory ChEL with I-II-III. 293 cells were transfected with empty vector or with plasmid DNAs encoding I-II-III $(0.5 \mu \mathrm{g})$ plus $1.5 \mu \mathrm{g}$ of either vector alone (I-II-III) or secretory ChEL with an appended myc epitope tag (ChEL-myc). All cells were pulse labeled for 30 minutes with ${ }^{35} \mathrm{~S}$-labeled amino acids and chased for the times shown in the presence of BFA $(5 \mu \mathrm{g} / \mathrm{ml})$. The cells were lysed and immunoprecipitated either anti-Tg (lanes 1-7) or anti-myc (lanes $8-11)$. Immunoprecipitates were analyzed by nonreducing 5.5\% SDSPAGE and fluorography, with the I-II-III region shown. The I-II-III band seen at time 0 appears equivalent to that of the $D$ isoform, while ChELmyc favorably coprecipitates a faster-migrating band equivalent to that of the mature $\mathrm{E}$ isoform (arrow). The position of a 176-kDa molecular mass marker is shown at left.

been more elusive. In spite of this, it appears that most congenital hypothyroidism with deficient $\mathrm{Tg}$ is caused by mutations in which intracellular Tg transport is blocked, rather than by defects in the ability to synthesize thyroid hormone per se $(1,32)$. Evolutionary pressure definitely exists for $\mathrm{Tg}$ to be maintained as a secreted protein, as its inability to do so (at least in Merino sheep) compromises viability to a stage where animals cannot reach reproductive age
$(33,34)$. We now present what we believe to be the first evidence directly implicating the ChEL domain in assisting the intracellular protein transport of $\mathrm{Tg}$.

One way in which the ChEL domain may participate in Tg export is by facilitating Tg folding. We previously suspected that ChEL forms an independently folded domain within $\mathrm{Tg}$, because its replacement by authentic acetylcholinesterase results in a chimera with active cholinesterase function (26). Yet single point mutations in the ChEL region appear sufficient to prevent formation of disulfide bonds that are made in upstream $\mathrm{Tg}$ regions, as evidenced not only by remarkable reactivity with AMS (Figure 1), but also by direct assessment of global disulfide maturation (with $\operatorname{cog}$ and $r d w$ mutants being trapped in partially oxidized folding intermediates; Figure 2). Using a cleavable signal peptide, we now find that the isolated ChEL domain is a very effective secretory protein in its own right (Figure 4), whereas I-II-III alone cannot escape ER retention (Figure 5, A and B). The simplest hypothesis (still to be tested) is that exposed chaperone-binding regions on Tg I-II-III confers its ER retention. Remarkably, our current evidence supports that the presence of the ChEL domain in the ER enriches for an oxidatively mature form of I-II-III (Figure 8), increases intracellular recovery of I-II-III (Figures 7 and 10), and allows I-II-III to be secreted (Figure 5C) in a form that is both endoglycosidase $\mathrm{H}$ resistant (data not shown) and appropriately oxidized (Figure 9). Thus, we propose that as long as the ChEL domain is itself properly folded (Figure 11B), it functions as an intramolecular chaperone within the context of full-length $\mathrm{Tg}$, facilitating the efficiency of "on-pathway" protein folding, leading to ER exit.

However, this does not appear to be the whole story, because the ChEL domain is transported with Tg out of the ER. Surprisingly, this is not simply because the ChEL domain is physically contigu-
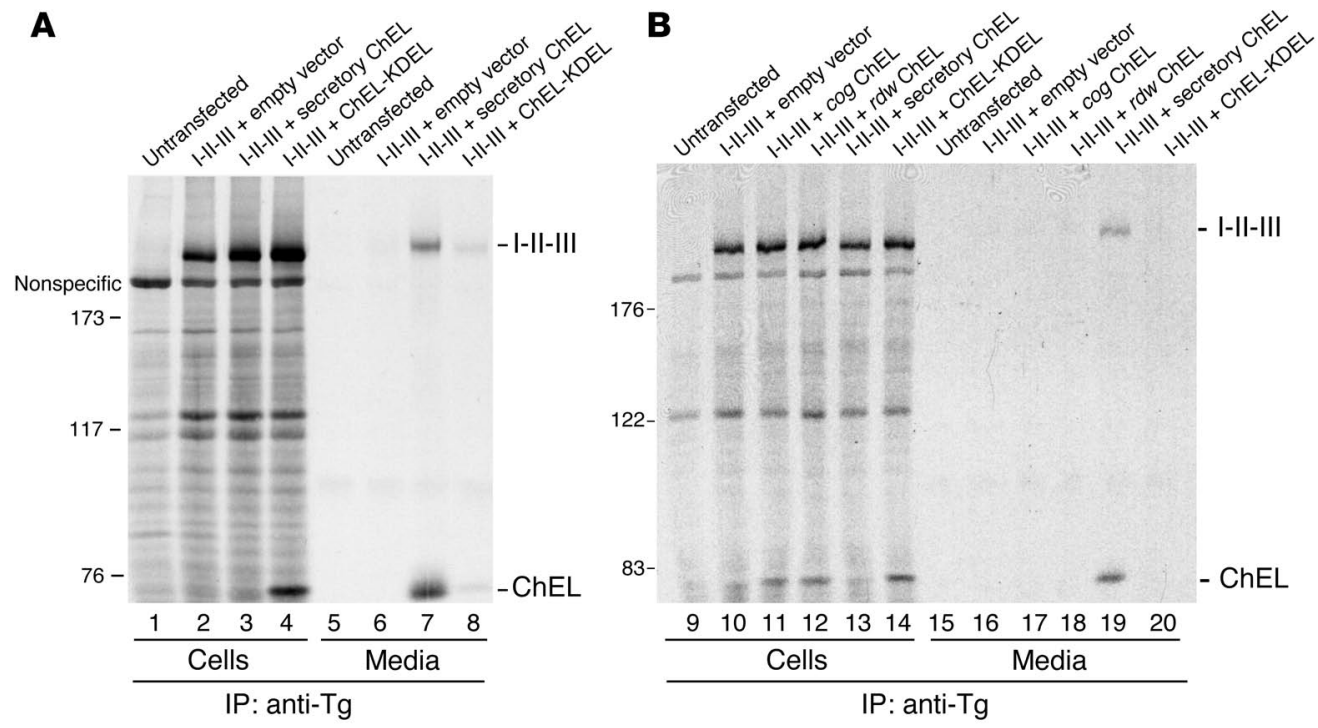

\section{Figure 11}

The Tg ChEL domain functions as a molecular escort. (A) 293 cells were untransfected or transiently cotransfected with $0.5 \mu \mathrm{g}$ plasmid DNA encoding I-II-III plus $2.5 \mu \mathrm{g}$ of the constructs indicated. The cells were pulse labeled for 30 minutes with ${ }^{35} \mathrm{~S}$-labeled amino acids and chased in complete medium for 4 hours. Cell lysates and media were immunoprecipitated with anti-Tg and analyzed by SDS-PAGE and fluorography. Note that anti-Tg recognizes both I-II-III and the ChEL protein; the ChEL-KDEL construct is selectively retained intracellularly, while secretory ChEL is released to the medium. Intracellular retention of ChEL-KDEL causes a parallel retention of I-II-III. (B) Cells cotransfected and pulse labeled as in $\mathbf{A}$ were chased for 5 hours before analysis as above. Note that the secretory ChEL domain bearing the cog or rdw mutation cannot support the secretion of I-II-III. The positions of molecular mass markers are shown at left. 
ous within the $\mathrm{Tg}$ protein. Indeed, secretory ChEL, made as a separate protein, is engaged in a direct physical interaction with I-II-III throughout the secretory pathway and into the extracellular space (Figure 6A). Nevertheless, most newly synthesized I-II-III does not exit the ER with a secretory ChEL partner that was synthesized during the same pulse-labeling period. Rather, the kinetics of I-II-III secretion are delayed in comparison to the speedy (and efficient) release of coexpressed secretory ChEL (Figure 5C). Evidently, intracellular transport of I-II-III must wait for subsequently translated ChEL domain to become available for interaction (Figure 6B). Thus, in all likelihood, I-II-III must undergo some preliminary folding prior to its interaction with ChEL. Indeed, the ChEL domain has an even stronger affinity for well-folded I-II-III than it does for immature I-II-III (Figure 10). This binding is not accidental but essential to the Tg secretory process. Specifically, retention of ChEL within the ER-Golgi system via an engineered KDEL sequence co-retains the mature I-II-III (Figure 11) - even as it permits the ChEL domain to function normally as a molecular chaperone (Figure 8 ). Thus, we are forced to conclude that the ChEL domain also functions as a molecular escort (3) required to convey Tg I-II-III out of the ER.

It is interesting to consider the evolutionary dependence of $\mathrm{Tg}$ on these mechanisms of intramolecular rescue, given that the major thyroid hormone-forming site in $\mathrm{Tg}$ is at its extreme amino terminus (35). One might predict that a highly truncated $\mathrm{Tg}$ with fewer disulfide bonds and preservation of crucial amino acids for thyroid hormone formation $(10,11)$ would allow survival without the need of intramolecular chaperone or escort functions. Indeed, there is speculation that such a short aminoterminal portion of $\mathrm{Tg}$ may be all that is required for human survival, which requires ongoing thyroid hormone synthesis (36). However, evolutionary and environmental circumstances (i.e., iodide unavailability on the earth's crust) have promoted development of a more complex Tg structure for iodide storage in vertebrate organisms (1).

In summary, we propose that intracellular Tg transport for thyroid hormone synthesis is dependent on intramolecular chaperone and escort functions embedded within its ChEL domain.

\section{Methods}

Materials. AMS, Lipofectamine 2000, FBS, penicillin, and streptomycin were from Invitrogen; Complete Protease Inhibitor Cocktail was from Roche; BFA, protein $\mathrm{G}$-agarose, and protein A-agarose were from Sigma-Aldrich; endoglycosidase $\mathrm{H}$ was from New England Biolabs Inc.; Trans ${ }^{35} \mathrm{~S}$-Label was from MP Biomedicals; Zysorbin was from Zymed Laboratories Inc. (Invitrogen); TransIT-LT1 Transfection Reagent was from Mirus. Rabbit polyclonal antimyc was from Immunology Consultants Laboratory Inc. and monoclonal anti-acetylcholinesterase (MAB303) was from Millipore. Rabbit polyclonal anti- $\mathrm{Tg}$ (containing antibodies against epitopes at both $\mathrm{N}$ - and C-terminal regions of the protein) has been previously described (14).

Site-directed mutagenesis of mouse $\mathrm{Tg}$ cDNA. ChEL domain mutations were introduced with the QuikChange Site-Directed Mutagenesis Kit (Stratagene) using the following mutagenic primers: ChEL-KDEL (5'-GCCTGTCCCCAAGAGCTACAGCAAAGACGAGCTATAGGCCGCTTCCCTTTAGTGAGGG-3' and 5'-CCCTCACTAAAGGGAAGCGGCCTATAGCTCGTCTTTGCTGTAGCTCTTGGGGACAGGC-3'); ChEL-myc (5'-CAAGAGCTACAGCAAAGAACAGAAACTGATCTCTGAGGAGGACTTATGATTAATGCTTCG-3' and 5'-CGAAGCATTAATCATAAGTCCTCCTCAgagatCAGTtTCTGTTCTTTGCTGTAGCTCTTG-3'). The trun- cated $\mathrm{Tg}$ region I-II-III was made by introducing a stop codon at L2169 (5'-GGAAGTCTGGAATCCCTTAGGTCCAATCTGATGTAACATCC-3' and 5'-GGATGTTACATCAGATTGGACCTAAGGGATTCCAGACTTCC-3') within Tg subcloned into pCMS (BD Biosciences - Clontech). To make the secretory ChEL domain, a Sal I restriction site was introduced into the $5^{\prime}$ end of ChEL by using a pair of mutagenesis primers (5'-CCTACATCTACCGGAAGTCTGGTCGACCTTTGGTCCAATCTGATGTAACATCC-3' and 5'-GGATGTTACATCAGATTGGACCAAAGGTCGACCAGACTTCCGGTAGATGTAGG-3') with subcloning into the SalI and NotI sites of pCMS. Each mutation was confirmed by direct cDNA sequencing before expression in 293 cells. The prolactin signal peptide in PSPPS1 (a kind gift from V. Lingappa, UCSF, San Francisco, California, USA) was fused in frame at the 5 ' end of the ChEL cDNA using NheI and SalI sites.

Cell culture and transfection. 293 cells were cultured in DMEM with 10\% FBS in 6-well plates at $37^{\circ} \mathrm{C}$ in a humidified $5 \% \mathrm{CO}_{2}$ incubator. Plasmids were transiently transfected using TransIT-LT1 or Lipofectamine 2000 transfection reagent according to the manufacturer's instructions.

Metabolic labeling and immunoprecipitation. Transfected 293 cells were starved for 30 minutes in Met/Cys-free DMEM, then pulse labeled with $180 \mu \mathrm{Ci} / \mathrm{ml}^{35} \mathrm{~S}$-labeled amino acids for 30 minutes. The cells were then washed with an excess of cold Met/Cys and chased in complete DMEM. At each time point, cells were lysed in buffer containing $1 \%$ NP-40, $0.1 \%$ SDS, $0.1 \mathrm{M} \mathrm{NaCl}, 2 \mathrm{mM}$ EDTA, $25 \mathrm{mM}$ Tris $\mathrm{pH}$ 7.4, and protease inhibitor cocktail (for cells expressing authentic acetylcholinesterase, lysis buffer contained 1\% Triton X-100, 0.1 M NaCl, 2 mM EDTA, $25 \mathrm{mM}$ Tris $\mathrm{pH} 7.4$, and protease inhibitors). Where indicated, $20 \mathrm{mM} \mathrm{N}$-ethylmaleimide was also added to the lysis buffer. For immunoprecipitation, anti-Tg or anti-acetylcholinesterase antibodies were incubated with cell or media samples overnight at $4^{\circ} \mathrm{C}$, and the immunoprecipitate was recovered with protein Aagarose (for anti-Tg) or protein $\mathrm{G}$-agarose (for anti-acetylcholinesterase). For coimmunoprecipitation studies, samples were incubated overnight at $4{ }^{\circ} \mathrm{C}$ with anti-myc antibodies and protein A-agarose. Immunoprecipitates (or coprecipitates) were washed 3 times before being boiled in SDS sample buffer with or without reducing agent, resolved by SDS-PAGE, and analyzed by fluorography or phosphorimaging.

Alkylation of $\mathrm{Tg}$ Cys thiols. Immunoprecipitated $\mathrm{Tg}$ was resuspended and incubated in a buffer containing $2 \%$ SDS, $50 \mathrm{mM}$ Tris $\mathrm{pH} 7.4$ with or without $5 \mathrm{mM}$ AMS for 1 hour at $30^{\circ} \mathrm{C}$. The reaction was stopped by boiling in SDS sample buffer plus $0.1 \mathrm{M}$ DTT before analysis by SDS-PAGE.

Endoglycosidase H digestion. Immunoprecipitates were boiled for $10 \mathrm{~min}$ utes in $0.5 \%$ SDS containing 1\% 2-mercaptoethanol in $20 \mathrm{mM}$ Tris $\mathrm{pH} 7.4$ and digested with $250 \mathrm{U}$ of endoglycosidase $\mathrm{H}$ in $50 \mathrm{mM}$ sodium citrate, $\mathrm{pH} 5.5$, for 1 hour at $37^{\circ} \mathrm{C}$.

\section{Acknowledgments}

This work was supported by NIH grant DK40344 (to P. Arvan). We thank members of the Arvan laboratory, especially Xiang Zhao, for helpful suggestions during the course of this work.

Received for publication January 28, 2008, and accepted in revised form May 21, 2008.

Address correspondence to: Peter Arvan, Division of Metabolism, Endocrinology and Diabetes, University of Michigan, 5560 MSRB2, 1150 W. Medical Center Drive, Ann Arbor, Michigan 48109-0678, USA. Phone: (734) 936-5006; Fax: (734) 936-6684; E-mail: parvan@umich.edu.
1. Di Jeso, B., and Arvan, P. 2004. Thyroglobulin structure, function, and biosynthesis. In The thyroid. L.E. Braverman, and R. Utiger, editors. Lippincott
Williams \& Wilkins. Philadelphia, Pennsylvania, USA. 77-95.

2. den Hartog, M.T., Sijmons, C.C., Bakker, O., Ris-
Stalpers, C., and de Vijlder, J.J. 1995. Importance of the content and localization of tyrosine residues for thyroxine formation within the $\mathrm{N}$-terminal 
part of human thyroglobulin. Eur. J. Endocrinol. 132:611-617

3. Kim, P.S., and Arvan, P. 1998. Endocrinopathies in the family of endoplasmic reticulum (ER) storage diseases: disorders of protein trafficking and the role of ER molecular chaperones. Endocr. Rev. 19:173-202.

4. Arvan, P., et al. 1997. Intracellular protein transport to the thyrocyte plasma membrane: potential implications for thyroid physiology. Thyroid. 7:89-105.

5. Muresan, Z., and Arvan, P. 1997. Thyroglobulin transport along the secretory pathway. Investigation of the role of molecular chaperone, GRP94, in protein export from the endoplasmic reticulum. J. Biol. Chem. 272:26095-26102.

6. Veneziani, B.M., Giallauria, F., and Gentile, F. 1999. The disulfide bond pattern between fragments obtained by the limited proteolysis of bovine thyroglobulin. Biochimie. 81:517-525.

7. Schumacher, M., et al. 1986. Primary structure of Torpedo californica acetylcholinesterase deduced from its cDNA sequence. Nature. 319:407-409.

8. Swillens, S., Ludgate, M., Mercken, L., Dumont, J.E. and Vassart, G. 1986. Analysis of sequence and structure homologies between thyroglobulin and acetylcholinesterase: possible functional and clinical significance. Biochem. Biophys. Res. Comm. 137:142-148.

9. Mori, N., Itoh, N., and Salvaterra, P.M. 1987. Evolutionary origin of cholinergic macromolecules and thyroglobulin. Proc. Natl. Acad. Sci. U. S. A. 84:2813-2817.

10. Marriq, C., Lejeune, P.J., Venot, N., and Vinet, L. 1991. Hormone formation in the isolated fragment 1-171 of human thyroglobulin involves the couple tyrosine 5 and tyrosine 130 . Mol. Cell. Endocrinol. 81:155-164.

11. Dunn, A.D., Corsi, C.M., Myers, H.E., and Dunn, J.T. 1998. Tyrosine 130 is an important outer ring donor for thyroxine formation in thyroglobulin. J. Biol. Chem. 273:25223-25229.

12. van de Graaf, S.A., et al. 2001. Up to date with human thyroglobulin. J. Endocrinol. 170:307-321.

13. Kim, P., Bole, D., and Arvan, P. 1992. Transient aggregation of nascent thyroglobulin in the endoplasmic reticulum: relationship to the molecular chaperone, BiP. J. Cell Biol. 118:541-549.

14. Kim, P.S., Kwon, O.-Y., and Arvan, P. 1996. An endoplasmic reticulum storage disease causing congenital goiter with hypothyroidism. J. Cell Biol. 133:517-527.

15. Baryshev, M., et al. 2004. Unfolded protein response is involved in the pathology of human congenital hypothyroid goiter and rat non-goitrous congenital hypothyroidism. J. Mol. Endocrinol. 32:903-920.

16. Menon, S., et al. 2007. Oxidoreductase interactions include a role for ERp72 engagement with mutant thyroglobulin from the rdw/rdw rat dwarf. J. Biol. Chem. 282:6183-6191.

17. Caputo, M., et al. 2007. Congenital hypothyroidism with goitre caused by new mutations in the thyroglobulin gene. Clin. Endocrinol. (Oxf.). 67:351-357.

18. Pardo, V., et al. 2007. Congenital hypothyroidism due to a mutant thyroglobulin $(\mathrm{Tg})$ retained in the endoplasmatic reticulum: a functional analysis of the novel mutation $(6701 \mathrm{C}>\mathrm{A} ; \mathrm{A} 2215 \mathrm{D})$ in the TG gene. Presented at the 78th Annual Meeting of the American Thyroid Association. October 4-7. New York, New York, USA

19. Caron, P., et al. 2003. Compound heterozygous mutations in the thyroglobulin gene (1143delC and $6725 \mathrm{G}->$ A [R2223H]) resulting in fetal goitrous hypothyroidism. J. Clin. Endocrinol. Metab. 88:3546-3553.

20. Kitanaka, S., et al. 2006. A novel compound heterozygous mutation in the thyroglobulin gene resulting in congenital goitrous hypothyroidism with high serum triiodothyronine levels. J. Hum. Genet. 51:379-382.

21. Rivolta, C.M., and Targovnik, H.M. 2006. Molecular advances in thyroglobulin disorders. Clin. Chim. Acta. 374:8-24.

22. Matakidou, A., et al. 2004. Risk of non-medullary thyroid cancer influenced by polymorphic variation in the thyroglobulin gene. Carcinogenesis. 25:369-373.

23. Kim, P.S., et al. 2000. A missense mutation G2320R in the thyroglobulin gene causes non-goitrous congenital primary hypothyroidism in the WIC-rdw rat. Mol. Endocrinol. 14:1944-1953.

24. Hishinuma, A., et al. 2000. A novel missense mutation (G2320R) in thyroglobulin causes hypothyroidism in rdw rats. Endocrinology. 141:4050-4055.

25. Kim, P.S., et al. 1998. A single amino acid change in the acetylcholinesterase-like domain of thyroglob- ulin causes congenital goiter with hypothyroidism in the $\operatorname{cog} / \operatorname{cog}$ mouse: a model of human ER storage diseases. Proc. Natl. Acad. Sci. U. S. A. 95:9909-9913.

26. Park, Y.N., and Arvan, P. 2004. The acetylcholinesterase-homology region is essential for normal conformational maturation and secretion of thyroglobulin. J. Biol. Chem. 279:17085-17089.

27. Frand, A.R., and Kaiser, C.A. 1999. Ero1p oxidizes protein disulfide isomerase in a pathway for disulfide bond formation in the endoplasmic reticulum. Mol. Cell. 4:469-477.

28. Di Jeso, B., et al. 2005. Mixed-disulfide folding intermediates between thyroglobulin and ER resident oxidoreductases ERp57 and PDI. Mol. Cell. Biol. 25:9793-9805.

29. Kim, P.S., and Arvan, P. 1991. Folding and assembly of newly synthesized thyroglobulin occurs in a preGolgi compartment. J. Biol. Chem. 266:12412-12418.

30. Morel, N., Leroy, J., Ayon, A., Massoulie, J., and Bon, S. 2001. Acetylcholinesterase $\mathrm{H}$ and $\mathrm{T}$ dimers are associated through the same contact. Mutations at this interface interfere with the C-terminal $\mathrm{T}$ peptide, inducing degradation rather than secretion. J. Biol. Chem. 276:37379-37389.

31. Ekholm, R. 1990. Biosynthesis of thyroid hormones. Int. Rev. Cytol. 120:243-288.

32. Vono-Toniolo, J., et al. 2005. Naturally occurring mutations in the thyroglobulin gene. Thyroid. 15:1021-1033.

33. Falconer, I.R. 1966. Studies of the congenitally goitrous sheep. The iodinated compounds of serum, and circulating thyroid-stimulating hormone. Biochem. J. 100:190-196.

34. de Vijlder, J.J. 2003. Primary congenital hypothyroidism: defects in iodine pathways. Eur. J. Endocrinol. 149:247-256.

35. Dunn, J.T., and Dunn, A.D. 1999. The importance of thyroglobulin structure for thyroid hormone biosynthesis. Biochimie. 81:505-509.

36. van de Graaf, S.A., et al. 1999. A premature stop codon in thyroglobulin messenger RNA results in familial goiter and moderate hypothyroidism. J. Clin. Endocrinol. Metab. 84:2537-2542.

37. Kim, P.S., et al. 2008. Defective protein folding and intracellular retention of thyroglobulin-R19K mutant as a cause of human congenital goiter. Mol. Endocrinol. 22:477-484. 